\title{
Spatial Expansion Analysis in Yantai Urban Agglomeration with RS and GIS
}

\author{
Yun-hua Sun ${ }^{1,2}$, Ximin Cui ${ }^{1}$,Song Tian ${ }^{1}$ \\ 1. College of Geoscience and Surveying Engineering, \\ China University of Mining \&Technology, \\ Beijing, China. \\ yunhua07@163.com
}

\begin{abstract}
Cities and surrounding suburbs are Earth's fastest growing land use.For the purpose of predicting future urban evolution trends and elaborating rational policies for regulation and control, it's significant to distinguish correctly the rules of urban expansion and to analyze the evolution process. Offering a variety of data of urban expansion, the earth observation technology by remote sensing becomes the main approach. Based on the TM image in 1989, ETM+ image in 2000 and TM image in 2009, we use RS and GIS to extract the boundary of urban entity of Yantai urban agglomeration and analyze the area, direction, type and rules of urban expansion from 1989 to 2009. It's indicated that expansion of Yantai urban agglomeration developed rapidly in recent 20 years, and greatly influenced by terrain, port-centered industry, traffic circulation and the government regulation.
\end{abstract}

Index Terms-RS; GIS; Urban agglomeration expansion.

\section{I.INTRODUCTION}

Urbanization is the physical growth of urban areas as a result of rural migration and even suburban concentration into cities, particularly the very largest ones ${ }^{[1]}$. It is a process that includes a wide range of social, demographic, spatial and economic transformation. Urbanization is the inevitable result of the social and economic development, but also the integral part of the socio-economic development and strong pusher. At present, the spatial expansion of urban land is increasingly becoming the main features of the land-use change and measuring index of urbanization level ${ }^{[2]}$.

There are many experts study urbanization expansion with remote sensing and geographic information system.

Liu Sheng-he, Wu Chuan-jun studied urban land usegrowth based on the land use maps of Beijing in 1982,

\author{
1992Weihong Cui ${ }^{2}$, TuoWang ${ }^{2}$, Liang $\mathrm{Yu}^{2}$ \\ 2. Institute of Remote Sensing Applications Chinese \\ Academy of Sciences. \\ Beijing, China \\ weihong.cui@gmail.com
}

and 1997, analyzed its spatial and temporal process of urbanland use growth through spatial-clustering and historical-morphological approaches ${ }^{[3]}$. He Chunyang, Chen Jin regarded that the city expansion was the result of the collective effects of exogenous constraints and internal expanding ability of urban pixel, and they developed a city expansion model in metropolitan area $(\mathrm{CEM})^{[4]}$. Joseph O. Sexton, Xiao-Peng Song developed an empirical method for retrieving annual, long-term continuous fields of impervious surface cover from the Landsat archive and applied it to the Washington, D.C.-Baltimore, MD megalopolis from 1984 to $2010^{[5]}$.

The above research have made studies on a variety of large and medium-sized urban expansion, but the research is still rare in expansion of urban agglomerations and extended difference among different counties. Since the reform and opening up, Yantai region, lay in the coastal zone of the Shandong Peninsula, its rapid economic development and the rapid urbanization process has produced a significant impact on regional land use and ecological environment. Therefore, we choose Yantai region as the study area, apply remote sensing technology as a means of access to information and use geographic information system technology as a means of information processing research, in order to analyze the area, direction, type and rules of urban expansion from 1989 to 2009 through different index calculation and further study Yantai extended-oriented laws.

\section{METHODS}

\section{A. Study area}

Our study area is Yantai urban agglomeration, lay in north of Shandong Peninsula, encompassing five districts 
(Zhifu, Laishan, Muping, Fushan, Development zones), seven counties (Qixia, Haiyang, Longkou, Laizhou, Zhaoyuan, Penglai and Changdao), which east longitude is $119^{\circ} 34^{\prime} \sim 121^{\circ} 57^{\prime}$, northern latitude is $36^{\circ} 16^{\prime} \sim 38^{\circ} 23^{\prime}$, total area is $13748 \mathrm{~km}^{2}$ (Figure 1). From Yantai census in 2010, we found that the human population was $6.96 \times 10^{6}$.It is mountainous in this region. The main river is Wulong River, Dagujia River, Xinan River and Jie River, belonging to peninsular edge river system and flowing to south and north. Thanks to convenient transportation, three-dimensional traffic is composed by sea transportation, railway, highway, aviation.

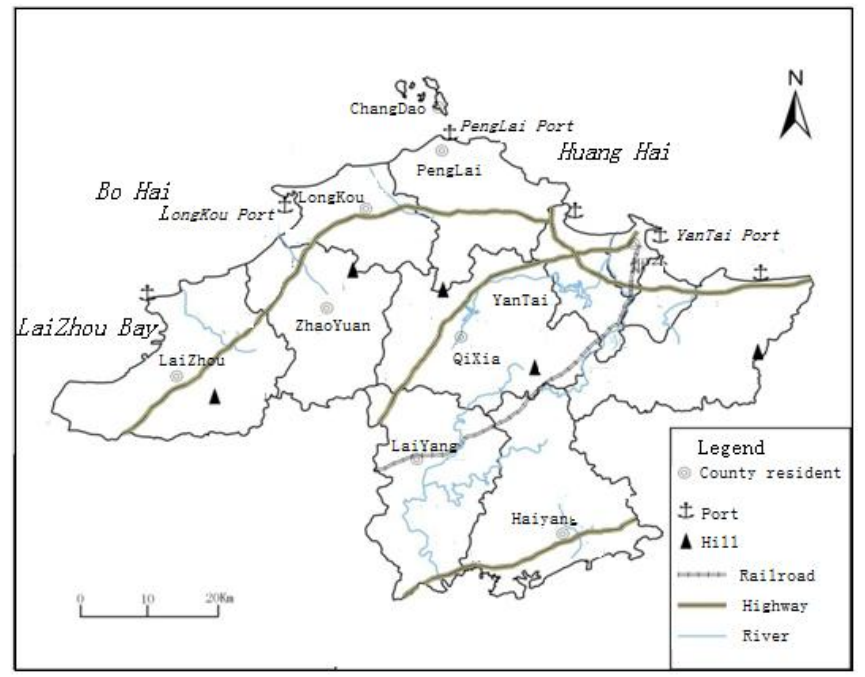

Fig.1 Location of Yantai Urban agglomeration

\section{B. Model}

We model area proportion of urban and rural $(P U)$, entity area of study urban $(U L)$ divided by the total land area of study area $(T L)$, and then multiplied by $100 \%$.

$$
P U=\frac{U L}{T L} \times 100 \%(1)
$$

In order to further study spatial changes, we built an urban expansion index, which computational formula was:

$$
E I=\frac{U L_{t+1}-U L_{t}}{T L} \times 100 \%
$$

Where EI means urban expansion index of study area, $U L_{t+1}$ represents urban entity area in $t+1$ period, while $U L_{t}$ is urban entity area in t period, TL is regarded as total area. Without considering time factor in this index, so, in order to compare changes between two periods, we use EI divided by interval years, that is to say, use annual average urban expansion index $(k)$ to analyze the data.

\section{Data and process}

We choose TM image in Jun.1989, ETM+ image in Jun.2000, TM image in Aug.2009 and topographic map with scale of 1:50000. The quality of images is very well and capable to make analyze. They represent three development phases in reform and opening respectively. It is possible to demonstrate urban development in different phase under the background of economic rapid growth. With the help of topographic map, we conduct geometric registration and conduct operation on three images and finally unify to Gaussian projection. Accuracy of geometric registration is controlled within one pixel. Then, we proceed to do the visual interpretation through arcgis9.3 to obtain spatial information and make sure extraction accuracy is more than $95 \%$. Here, in order to express classification result, we introduce the concept of urban entity boundary ${ }^{[6]}$. Its definition is continuous distribution of geographical entities in the space with the landscape characteristics of the city (settlement). Specifically, the city entities include urban built-up areaand the city boundary zone connected to the built-up area (rural-urban continuum). The water body and urban green space in inner city, is also part of the urban entity. Therefore, the outer edge of the urban entity is urban entity boundary. Then, we conduct spatial overlay analysis using GIS software, in order to get spatial and attribute data in different counties and cities of the urban agglomeration.

\section{RESULTS}

\section{A. Area proportion changes}

The entity area of different counties in Yantai region changed significantly among 20 years (Tab.1). Whole city entity area expanded from $101.75 \mathrm{~km}^{2}$ in 1989 to $426.87 \mathrm{~km}^{2}$ in 2009, which was more than four times. During this area changes, from 1989 to 2000 , expanded $97.27 \mathrm{~km}^{2}$, while from 2000 to 2009 , the number was $227.85 \mathrm{~km}^{2}$.

\section{B. Annual average urban expansion index changes}

The whole study area has been expanded quickly with annual average urban expansion index $(k)$ of $18.4 \%$, which is three times than previous period (Tab.2). Within the study area, Yantai City's expansion speed during two periods 
equals basically, that latter $k$ is higher $4.7 \%$ than previous one. Considering Changdao County, under the development of travel economic in 1989-2000, urban entity expanded toward north Changshan Island, resulting in $P U$ and $k$ higher two times than period of 2000-2009. Penglai County

Tab. 1 Contrast of urban entity and percentage in different periods

\begin{tabular}{|c|c|c|c|c|c|c|c|c|c|c|c|c|c|c|c|c|c|c|c|c|}
\hline \multirow{2}{*}{ year } & \multicolumn{2}{|c|}{ Changdao } & \multicolumn{2}{|c|}{ Haiyang } & \multicolumn{2}{|c|}{ Laiyang } & \multicolumn{2}{|c|}{ Laizhou } & \multicolumn{2}{|c|}{ Longkou } & \multicolumn{2}{|c|}{ Penglai } & \multicolumn{2}{|c|}{ Qixia } & \multicolumn{2}{|c|}{ Yantai } & \multicolumn{2}{|c|}{ Zhaoyuan } & \multicolumn{2}{|c|}{ Study area } \\
\hline & $U L$ & $P U$ & $U L$ & $P U$ & $U L$ & $P U$ & $U L$ & $P U$ & $U L$ & $P U$ & $U L$ & $P U$ & $U L$ & $P U$ & $U L$ & $P U$ & $U L$ & $P U$ & $U L$ & $P U$ \\
\hline 1989 & 0.96 & $1.85 \%$ & 3.48 & $0.19 \%$ & 6.61 & $0.43 \%$ & 6.5 & $0.33 \%$ & 15.16 & $1.8 \%$ & 7.48 & $0.58 \%$ & 4.27 & $0.21 \%$ & 51.22 & $1.99 \%$ & 6.07 & $0.44 \%$ & 101.75 & $0.75 \%$ \\
\hline 2000 & 2.57 & $4.58 \%$ & 7.35 & $0.39 \%$ & 9.04 & $0.52 \%$ & 9.34 & $0.5 \%$ & 21.32 & $2.39 \%$ & 17.42 & $1.44 \%$ & 4.92 & $0.24 \%$ & 115.35 & $4.36 \%$ & 11.71 & $0.82 \%$ & 199.02 & $0.87 \%$ \\
\hline 2009 & 3.21 & $5.72 \%$ & 32.1 & $1.7 \%$ & 36.48 & $2.11 \%$ & 36.07 & $1.92 \%$ & 74.16 & $8.3 \%$ & 25.73 & $2.28 \%$ & 10.76 & $0.53 \%$ & 176.69 & $6.6 \%$ & 28.67 & $2.0 \%$ & 426.87 & $3.1 \%$ \\
\hline
\end{tabular}

Tab.2 Urban expansion index (EI) and annual average urban expansion index (k) in different periods

\begin{tabular}{|c|c|c|c|c|c|c|c|c|c|c|c|c|c|c|c|c|c|c|c|c|}
\hline \multirow{2}{*}{ year } & \multicolumn{2}{|c|}{ Changdao } & \multicolumn{2}{|c|}{ Haiyang } & \multicolumn{2}{|c|}{ Laiyang } & \multicolumn{2}{|c|}{ Laizhou } & \multicolumn{2}{|c|}{ Longkou } & \multicolumn{2}{|c|}{ Penglai } & \multicolumn{2}{|c|}{ Qixia } & \multicolumn{2}{|l|}{ Yantai } & \multicolumn{2}{|c|}{ Zhaoyuan } & \multicolumn{2}{|c|}{ Study area } \\
\hline & $E I$ & $k$ & $E I$ & $k$ & $E I$ & $k$ & $E I$ & $k$ & $E I$ & $k$ & $E I$ & $k$ & $E I$ & $k$ & $E I$ & $k$ & $E I$ & $k$ & $E I$ & $k$ \\
\hline 1989-2009 & 4.02 & 0.2 & 1.52 & 0.08 & 1.72 & 0.09 & 1.57 & 0.08 & 6.6 & 0.33 & 1.62 & 0.08 & 0.32 & 0.02 & 4.72 & 0.24 & 1.58 & 0.08 & 2.36 & 0.12 \\
\hline 1989-2000 & 2.88 & 0.26 & 0.21 & 0.02 & 0.14 & 0.01 & 0.15 & 0.01 & 0.69 & 0.06 & 0.88 & 0.08 & 0.03 & 0 & 2.36 & 0.22 & 0.39 & 0.04 & 0.71 & 0.07 \\
\hline 2000-2009 & 1.14 & 0.13 & 1.31 & 0.15 & 1.58 & 0.18 & 1.42 & 0.16 & 5.91 & 0.66 & 0.73 & 0.08 & 0.29 & 0.03 & 2.36 & 0.26 & 1.18 & 0.13 & 1.66 & 0.18 \\
\hline
\end{tabular}

expanded degree in 1989-2000 is higher $15 \%$ than 2000-2009 ones, but index $k$ is approximately same. Longkou County is obvious at the two indexes in 2000-2009, which is closely related with rapid development of Nanshan Group and Longgang Street Office expansion toward south and east. The two indexes are roughly identical in Haiyang, Laiyang, Laizhou and Zhaoyuan County. Qixia County's expansion degree is the slowest, $P U$ is only $3 \%$ in $1989-2000$ while $k$ equals $0.3 \%$, as well as $P U$ is $29 \%$ in $2000-2009, k$ is $3.2 \%$, far below the whole expansion index of study area. This is related to terrain of low mountains and hills in Qixia County, which limited development in this area.

\section{Oriented rules of urban entity expansion}

\section{1) Terrain orientation}

Flat area is beneficial to develop of urban region where expansion degree is large, while hilly area is limited to growth so as to expanding slowly. Take Qixia as an example, northeast part is Anli reservoir, that lead to urban entity expansion has a feature of northeast-southwest axial development distribution.

\section{2) Port-centered industry orientation}

According to port-centered industry planning of Yantai city, six groups were built under the spatial growth strategy guideline, including Zhifu Bay Group, Xigang Group, Penglai Group, Longkou Group, Laizhou Group and Haiyang Group. All of these lead to the rapid development of urban entities in the port area.

\section{3) Arterial traffic orientation}

Urban land value depends on its locational accessibility to some extent, so mature marginal zone type of landscape city is distributed in the central city to other areas along the transportation routes. The study area has a marked feature of growth oriented to road in 1989-2009. In the urban agglomeration, there are many highways, such as Rong-Wu highway, Shen-hai highway, Yan-wei highway, Qing-wei highway and provincial road, railway, promoting urban entity to expand along arterial traffic.

\section{4) Government regulation orientation}

Government regulation plays an important role in urban growth, such as implement policies of Peninsular Blue Economic Zone Strategy, arrangement of port-centeredindustry, construction of arterial traffic, etc.

\section{CONCLUSION}

We choose area proportion of urban and rural (PU) and annual average urban expansion index $(\mathrm{k}) \mathrm{t}$ to analyze quantitatively the characters of changes in urban entity expansion. Results show that significant changes are took 
place in Yantai agglomeration. Expansion speed is different in temporal and spatial scales. Changdao County and Yantai City expanded quickly in 1989-2000 and Longkou County is the first order in 2000-2009.

Through the analysis of expansion speed and orientation in Yantai agglomeration in 20 years, we can see that the influence of terrain, port-centered industry, arterial traffic and government regulation on urban expansion and certain oriented rules for its expansion.

Based on multi-temporal and multi-spectral remote sensing images, we could trace, observe and analyze dynamic process in urban expansion. At the same time, combining with social-economy statistical data in Yantai, it is possible to make quantitative and qualitative analysis of urban growth for purpose of providing useful, reliable reference data for planning department, finally boosting sustainable development of Shandong Peninsular northern coastal urban system.

\section{ACKNOWLEDGMENT}

Primary funding was provided by Foundation Item: National Natural Science Foundation of China, No. 71150001.Landsatdata was acquired from the USGS EROS Data Center.

\section{REFERENCES}

[1] Chen Ben-qing,Xu Han-qiu. Urban Expansion And Its Driving Force Analysis Using Remote Sensed Data_-A Case Of Xiamen City[J]. Economic Geography, 2005,25(1):79-83.

[2] Zeng Lei, Zong Yong, Lu Qi. Spatial-Temporal Feature of Urban Land Extension in Baoding City[J]. Resources Science,2004,26(4):96-103.

[3] Liu Sheng-he, Wu Chuan-jun, ShenHongquan. A GIS based Model of Urban Land Use Growth in Beijing[J]. ACTA GEOGRAPHICA SINICA, 2000,55(4):407-416.

[4] HeChunyang, Chen Jin, Shi Peijun, Fan Yida. City Expansion Model of Metropolitan Area in China: a Case Study of Beijing[J].ACTAGEOGRAPHICA

SINICA,2003,58(2):294-304.

[5] Joseph O. Sexton, Xiao-Peng Song, Chengquan Huang SaurabhChannan,Matthew E. Baker and John R. Townshend. Urban growth of the Washington, D.C.-Baltimore, MD metropolitan region from 1984 to 2010 by annual, Landsat-based estimates of impervious cover[J]. Remote Sensing of Environment, 2012,129, 42-53.

[6] FanZuojiang, Cheng Jicheng, Li Qi. Urban expansion research with combination of RS and GIS[J]. REMOTE SENSING INFORMATION, 1997,(3):12-16. 